\title{
Screening for atrial fibrillation in primary care
}

\author{
N M Wheeldon, D I Tayler, E Anagnostou, D Cook, C Wales, G D G Oakley
}

\begin{abstract}
Objective-To investigate a population of elderly people for atrial fibrillation and to determine how many of the cases identified might benefit from treatment with anticoagulants.

Methods-From a practice of four primary care physicians, 1422 patients aged 65 years and over were identified, of whom 1207 (85\% of the total population) underwent electrocardiographic screening to detect the presence of atrial fibrillation. Patients with the arrhythmia were further evaluated by echocardiography and interview, to stratify their risk of stroke based on echocardiographic and clinical risk factors, their perceived risk from anticoagulation, and their attitude towards this treatment. Their primary care physician was also interviewed to determine the factors influencing the prescription of anticoagulants.
\end{abstract}

Results-The arrhythmia occurred in 65 patients $(5.4 \%$ overall), its prevalence increasing markedly with age $(2.3 \%$ in 65 to 69 years age group; $8.1 \%$ in those over 85 ). Warfarin was being prescribed to $21.4 \%$ of these patients, although the findings of the study indicate that a further $20 \%$ were eligible for this treatment. Symptoms suggestive of cardiac failure were common $(32.1 \%)$ and coexisting pathology was often identified by cardiac ultrasound in these patients (left ventricular hypertrophy, $32.1 \%$; impaired left ventricular contractility, 21.4\%; left atrial dilatation, $80.4 \%$; mitral annular calcification, $42.9 \%$; mitral stenosis, $7.1 \%$; mitral regurgitation, $48.2 \%$; aortic stenosis, $8.9 \%$ ). In all but one case, the decision to anticoagulate was based on the clinical rather than the echocardiographic findings.

Conclusions-Individual risk-benefit assessment in elderly patients with atrial fibrillation suggests that almost half (41.4\%) are eligible for full anticoagulation with warfarin, whereas presently only one fifth are receiving this treatment. The decision to anticoagulate can be made on clinical grounds in most cases. If these results are confirmed, a doubling of the current number of patients taking anticoagulants can be anticipated.

(Heart 1998;79:50-55)

Keywords: atrial fibrillation; primary care; anticoagulant treatment

No less than seven major randomised clinical trials have now been reported that have evaluated the effect of anticoagulant treatment on the risk of thromboembolic events in patients with atrial fibrillation. ${ }^{1}$ Six of these trials were terminated prematurely on ethical grounds, as evidence emerged of a clinically and statistically significant benefit from treatment with warfarin. Although the data supporting the use of warfarin for this indication are compelling, the patients studied in these trials were very highly selected. Exclusion rates of up to $93 \%$ are reported, ${ }^{23}$ and even after entry into the studies, withdrawal from warfarin treatment reached $31 \% .^{2}$ Most exclusions resulted from refusal of anticoagulant treatment by the patient or their physician, mainly because of a perceived excess risk of bleeding complications. This selection process has led to the benefits and risks of warfarin being evaluated predominantly in a low risk group. Serious questions therefore have to be asked as to what extent these results can be extrapolated to the general population.

Epidemiological data suggest that atrial fibrillation is present in $0.4 \%$ of the population overall, although a striking relation exists with age, and prevalence rates of over $10 \%$ have been reported in the very old. ${ }^{4-9}$ Atrial fibrillation is therefore largely a disease of the elderly. These patients are at the highest risk of stroke and hence potentially stand to gain the most from prophylactic anticoagulants. They also present the greatest challenge as far as anticoagulation is concerned. Coexisting medical problems, degenerative brain disease, concomitant drug treatment, recurrent falls, and difficulties with compliance and monitoring are common in this population, and all add to the potential risks of warfarin treatment. It is therefore perhaps not surprising that this is the group physicians appear most reluctant to treat. ${ }^{10} 11$

Applying the results of the major trials to the population at large has enormous implications in terms of workload, resource allocation, and cost. $^{12}$ The large trials have provided information on overall risks and benefits in selected populations, but clinicians have to address these issues in an individual patient. At present, although we have estimates of the prevalence of atrial fibrillation and good evidence of a treatment benefit from warfarin in the trial subjects, there are no data on the number of "warfarin eligible" patients in the community. This information is essential for estimating the likely impact of the trial results on clinical practice, the resources required for their implementation, and whether screening for atrial fibrillation would be appropriate. Data of this type can only be obtained by investigating the whole of a population of patients with atrial fibrillation on an individual basis, which was the aim of the present study. 
We undertook electrocardiographic screening of the whole population aged 65 years and over from a primary care practice, to identify patients with atrial fibrillation and to estimate the prevalence of this condition in our local community. The subgroup of patients with atrial fibrillation was then further evaluated by cardiac ultrasound and interview in an attempt to stratify their risk of stroke, their risk from anticoagulation with warfarin, and their attitude towards this treatment. In this way, a profile of each individual patient's perceived risk and benefit was obtained, which was used to determine eligibility for treatment with anticoagulants. These profiles were then presented to the primary care physician, who was interviewed to determine the factors that influenced the prescription of anticoagulants. On the basis of this information, we were able to determine the number of warfarin eligible patients in the community who were currently untreated and estimate the increase in resources required if they were to be treated.

\section{Methods}

All patients aged 65 years and over from a single city practice of four primary care physicians were identified from a computerised patient database. All were contacted by post and invited to attend the surgery for a 12 lead electrocardiogram (ECG). In order to maximise patient numbers, non-responders were contacted again by letter and in the event of a further lack of response, by telephone or by a visit from their primary care physician. Patients were advised that the ECG was being performed as part of a research study. They were further advised that in the event of atrial fibrillation being present, they would be invited to attend for an echocardiogram and interview, and that the issue of anticoagulation would be discussed with themselves and their doctor. The vast majority of the ECGs were performed by a visiting ECG technician at the practice surgery, although domiciliary recordings were made available in selected cases. A full 12 lead recording was performed in each case and was interpreted by a hospital cardiologist. Patients found to be in sinus rhythm were thanked for their cooperation, but were not evaluated further at this stage. Those with atrial fibrillation were asked to attend the hospital for further investigation. The primary care physician was informed of other significant ECG abnormalities (such as heart block) requiring further attention, but other ECG findings were not considered in the present study.

Cross sectional and Doppler echocardiography was performed by an experienced operator using a Hewlett-Packard Sonos 2500 cardiac ultrasound machine, with high quality imaging, colour flow, and continuous and pulsed wave Doppler capabilities (Hewlett-Packard, Camas, Washington, USA). Each patient underwent a comprehensive echocardiographic examination, although for the purposes of this study attention was directed particularly towards purported echocardiographic risk factors that have been suggested by other workers. ${ }^{13}$ Echocardiographic data were ana- lysed independently by a second experienced operator. Left ventricular mass was calculated using the method of Devereux. ${ }^{15}$ Body surface area was estimated from height and weight measurements and was used to calculate the left ventricular mass index (LVMI). Normal values of LVMI were regarded as $<134 \mathrm{~g} / \mathrm{m}^{2}$ for men and $<110 \mathrm{~g} / \mathrm{m}^{2}$ for women. Left ventricular ejection fraction was calculated from ventricular volumes estimated using the modified Simpson's rule, ${ }^{16}$ a normal value being regarded as $>55 \%$. Because of the presence of atrial fibrillation, this result was obtained from the mean of five cardiac cycles. The left atrial diameter was measured from $M$ mode recordings made in the parasternal long axis view. Left atrial diameter was regarded as normal $(<4.0 \mathrm{~cm})$, borderline $(4.0-4.5 \mathrm{~cm})$, or significantly enlarged $(>4.5 \mathrm{~cm})$. The left atrium was inspected for signs of spontaneous echo contrast or thrombus. The presence of mitral annular calcification was noted and defined subjectively as being mild, moderate, or severe. Cross sectional imaging with colour flow and spectral Doppler was used to examine all valves, with particular emphasis on the mitral valve. Valvar stenosis or regurgitation was classified as mild, moderate, or severe on the basis of semiquantitative data obtained from colour flow and continuous wave Doppler studies (see Results for individual valve lesions).

Each patient was interviewed with regard to proposed clinical risk factors for thromboembolism - that is, a history of hypertension, previous stroke or transient ischaemic attack, heart failure, or myocardial infarction. ${ }^{14} 1718$ Patients were also questioned about bleeding disorders and peptic ulceration, whether they were receiving treatment with non-steroidal anti-inflammatory drugs, digoxin, aspirin, or warfarin, and whether they had experienced symptoms of palpitations. For patients not already treated with warfarin, the risks and benefits of this treatment were explained, as was the need for regular monitoring. These patients were then asked whether they would be willing to take this treatment if it were offered to them.

At the end of the study the clinical and echocardiographic information obtained from each patient with atrial fibrillation was presented to the patients' primary care physicians, who were asked for their reasons for treating or not treating each individual patient with warfarin, and whether this decision was influenced by the study data made available on that patient. The decision on whether to treat an individual patient with warfarin was left entirely in the hands of the primary care physician.

\section{Results}

From a total practice population of 7526 patients, $1422(18.9 \%)$ were identified as being aged 65 years or over at the outset of the study. Of this target group of 1422 patients, 1207 $(84.8 \%)$ agreed to have a 12 lead ECG performed. Twenty three domiciliary ECGs 
Table 1 Prevalence of atrial fibrillation $(A F)$ in relation to age group

\begin{tabular}{llll}
\hline Age (years) & Total patients (n) & $A F(n)$ & $A F(\%)$ \\
\hline $65-69$ & 444 & 10 & 2.3 \\
$70-74$ & 390 & 16 & 4.1 \\
$75-79$ & 240 & 14 & 5.8 \\
$80-84$ & 188 & 12 & 6.4 \\
$85+$ & 160 & 13 & 8.1 \\
\hline
\end{tabular}

Table 2 Echocardiographic findings in patients with atrial fibrillation $(n=56)$

\begin{tabular}{lcc}
\hline & $n$ & $\%$ \\
\hline Increased LV mass & 18 & 32.1 \\
Reduced ejection fraction & 12 & 21.4 \\
Left atrial diameter & 11 & 19.6 \\
$\quad<4.0 \mathrm{~cm}$ & 15 & 26.8 \\
$4.0-4.5 \mathrm{~cm}$ & 30 & 53.6 \\
$\quad 4.5 \mathrm{~cm}$ & 24 & 42.9 \\
Mitral annular calcification & 14 & 25.0 \\
$\quad$ Mild & 8 & 14.2 \\
$\quad$ Moderate & 2 & 3.6 \\
$\quad$ Severe & 0 & 0 \\
Definite intracardiac thrombus & & \\
\hline
\end{tabular}

were performed ( $1.9 \%$ of total), the remainder being recorded in the practice.

Sixty five patients $(5.4 \%$ overall; $95 \%$ confidence interval $4.6 \%$ to $6.2 \%$ ) were found to have atrial fibrillation. This included one patient with a permanent (VVI) pacemaker, known to be in atrial fibrillation, whose ECG revealed a paced rhythm and no evidence of atrial activity. A striking relation was found between the prevalence of atrial fibrillation and increasing age, ranging from $2.3 \%$ in the 65 to 69 years age group to $8.1 \%$ in patients over the age of 85 (table 1$)$. In five patients $(0.4 \%$ of the total screened), clinical findings and evidence from other ECGs taken before and after the study suggested that the arrhythmia was paroxysmal. Of the 65 patients with atrial fibrillation, nine ultimately refused to attend for echocardiography (generally due to ill health), leaving a total of 56 who underwent full evaluation.

\section{ECHOCARDIOGRAPHIC FINDINGS}

Echocardiographic findings are shown in table 2. Left ventricular mass index was increased in 18 patients $(32.1 \%)$ and the left ventricular ejection fraction reduced in $12(21.4 \%)$.

Three patients had evidence of a regional wall motion abnormality in keeping with previous infarction, despite an overall normal ejection fraction. Left atrial size was significantly enlarged in 30 (53.6\%), borderline in 15 $(26.8 \%)$, and normal in $11(19.6 \%)$.

Mitral annular calcification was noted in 24 patients $(42.9 \%)$ and was considered mild in 14 , moderate in eight, and severe in two. Four patients $(7.1 \%)$ had mitral stenosis (one previously unknown), which was regarded as mild in three cases (derived valve areas $1.7-2.0 \mathrm{~cm}^{2}$; one bioprosthesis) and moderate (derived valve area $1.4 \mathrm{~cm}^{2}$ ) in one case. One patient had a metallic mitral prosthesis which was functioning normally. Mitral regurgitation was demonstrated in 27 cases $(48.2 \%)$, being trivial $/$ mild in $14(25.0 \%)$, and of mild to moderate severity in $13(23.2 \%)$.

Spontaneous echo contrast in the left atrium was definitely seen in only two patients, but this
Table 3 Findings from the clinical history of patients with atrial fibrillation $(n=56)$

\begin{tabular}{lrr}
\hline & $n$ & $\%$ \\
\hline Hypertension & 10 & 17.9 \\
Stroke/TIA & 4 & 7.1 \\
Myocardial infarction & 6 & 10.7 \\
Cardiac failure & 18 & 32.1 \\
Palpitations & 18 & 32.1 \\
Rheumatic fever & 3 & 5.4 \\
Warfarin & 12 & 21.4 \\
Aspirin & 23 & 41.1 \\
Digoxin & 34 & 60.7 \\
NSAIDs & 8 & 14.3 \\
Bleeding contraindications & 2 & 3.6
\end{tabular}

TIA, transient ischaemic attack; NSAIDs, non-steroidal antiinflammatory drugs.

finding was highly subjective and dependent on ultrasound gain settings. Overall, this was felt to be an unreliable feature of the transthoracic examination. Discrete intracardiac thrombus was not identified in any patient, although the left atrial appendage was, of course, not visualised.

Aortic stenosis was found in five patients ( $8.9 \%$, two unknown), being regarded as mild in two (derived gradients 20 and $25 \mathrm{~mm} \mathrm{Hg}$ ), moderate in two ( 36 and $48 \mathrm{~mm} \mathrm{Hg}$ ), and severe in one case $(85 \mathrm{~mm} \mathrm{Hg})$. Four cases of trivial/mild aortic regurgitation were found, three being in patients with coexisting aortic stenosis.

Other significant echocardiographic findings were as follows: pulmonary hypertension (> $45 \mathrm{~mm} \mathrm{Hg}$ systolic) in four cases, moderate/ severe tricuspid regurgitation (5), mild tricuspid stenosis (1), echocardiographic features of hypertrophic obstructive cardiomyopathy (1, known), and lipomatous hypertrophy of the interatrial septum (1).

\section{CLINICAL FINDINGS}

The clinical findings are summarised in table 3. A history of treated hypertension was found in 10 patients $(17.9 \%)$ and of previous stroke or transient ischaemic attack in four $(7.1 \%)$. Eighteen patients $(32.1 \%)$ were receiving treatment with angiotensin converting enzyme (ACE) inhibitors or loop diuretics for symptoms suggestive of cardiac failure, although a definite history of myocardial infarction was obtained in only six $(10.7 \%)$. A history of palpitations, suggestive of an awareness of the arrhythmia, was given by 18 patients $(32.1 \%)$ and three $(5.4 \%)$ gave a definite history of rheumatic fever.

Twelve patients $(21.4 \%)$ were receiving warfarin, $23(41.1 \%)$ aspirin, and $34(60.7 \%)$ digoxin treatment. Only two patients $(3.6 \%)$ gave any significant history of bleeding disorder or definite peptic ulceration, and eight (14.3\%) were receiving treatment with NSAIDs. Of the 44 patients not receiving warfarin, $32(72.7 \%)$ were willing to receive this treatment after the risks, benefits, and the need for monitoring had been explained.

PRIMARY CARE PHYSICIAN INTERVIEW

On review of the study data, the primary care physicians of the 12 patients receiving warfarin treatment were satisfied that this treatment was 
Table 4 Reasons given for primary care physician withholding warfarin $(n=27)$

\begin{tabular}{ll}
\hline & $n$ \\
\hline Patient wishes & 9 \\
Multiple medical problems & 3 \\
Dementia & 2 \\
Monitoring and compliance problems & 2 \\
Housebound & 2 \\
Frailty/extreme age & 2 \\
Recurrent anaemia & 1 \\
Subdural haematoma & 1 \\
Aortic aneurysm & 1 \\
Recurrent epistaxis & 1 \\
Recurrent falls & 1 \\
Epilepsy & 1 \\
Alcoholism & 1 \\
\hline
\end{tabular}

appropriate. The treatment was therefore not withdrawn from any patient as a result of the study findings.

In 13 of the 44 patients with atrial fibrillation who were not anticoagulated $(20 \%$ of the total group), both the primary care physician and the patient (after explanation) agreed that warfarin was indicated and the decision was made to start this treatment as a direct result of the study findings. In five of these cases the presence of atrial fibrillation had been newly diagnosed from the ECG screening, whereas in the remaining eight the attention of the primary care physician was drawn to the issue of anticoagulation by the results of the study. In 27 cases the primary care physician had decided against anticoagulation despite the fact that 18 of these patients agreed to this treatment (table 4). Four patients refused anticoagulation on the basis of bleeding risk and monitoring, despite their doctor recommending this treatment. Advanced age alone was not considered a strong reason to withhold warfarin treatment, although many of the very elderly had other associated contraindications.

In all except one case, the decision to anticoagulate was based primarily on clinical rather than echocardiographic criteria. The presence of the arrhythmia itself and the absence of contraindications to warfarin treatment were the decisive factor for both the primary care physician, rather than the associated echocardiographic findings.

\section{Discussion}

Our study showed a $5.4 \%$ overall prevalence of atrial fibrillation in our local elderly population, although only one fifth $(21.4 \%)$ of these patients were receiving thromboembolic prophylaxis with warfarin. Based on clinical findings, echocardiographic criteria, and the wishes of the patients and their primary care physician, we estimate from this study that a further $20 \%$ of these patients with atrial fibrillation have a risk-benefit profile favouring treatment with anticoagulants. Overall, this suggests that almost half $(41.4 \%)$ of all patients with the arrhythmia of this age are eligible for warfarin treatment-twice the presently treated number. This finding has major resource and cost implications, the benefits of treatment in terms of reduced thromboembolic events (predominantly stroke) having to be weighed against the burdens of treatment and monitoring, and the potential bleeding hazard of anticoagulant treatment.

Excluding patients with metallic mitral valve prostheses, $32 \%$ of patients attending our anticoagulant clinics are being treated for atrial fibrillation, and a twofold increase in this subgroup would be required to treat all eligible patients in the community. In addition to the marginal costs of treating these extra patients, there is the additional cost of treating the increase in number of warfarin related bleeding complications that would inevitably follow. It has been argued that the low bleeding rates found in the major trials (largely as a result of extreme patient selection and strict monitoring) are unlikely to be reproduced in the general population. However, with the results of many trials now available it appears that the risks of haemorrhage can be reduced by using low intensity anticoagulation (international normalised ratio 2.0 to 3.0 ) without loss of efficacy. ${ }^{1}$ This is an important factor in the cost-benefit equation, as we know that stroke prevention with warfarin is cost-effective if bleeding rates can be kept low. ${ }^{19}$

It also seems likely that the selection process in the large trials and the intention to treat analyses may well have underestimated the benefit of warfarin treatment; many patients at relatively high risk of stroke being excluded on grounds that most would now consider inappropriate. Patients at highest risk from stroke potentially stand to gain the most from anticoagulation and in this respect treatment of a less highly selected population may well produce more in the way of overall benefit, although probably at an increased cost from bleeding complications.

The aim of the physician should be to treat all those patients whose risk-benefit profile is favourable, drawing the line where risk exceeds benefit. It is clear that this can only be achieved by assessing individuals rather than populations. We used this approach to estimate the shortfall of patients in our study who were both likely to benefit and who could safely be anticoagulated based on their perceived bleeding risk. This difference represents the number of additional patients who can be effectively treated, without increasing the overall rate of bleeding complications.

Given that we have identified a deficiency in the number of patients being treated with anticoagulants, does this provide an adequate basis for recommending ECG screening for the arrhythmia? It could be argued that this is unnecessary as only five $(7.7 \%)$ of the 65 patients with atrial fibrillation in this study were newly diagnosed by ECG screening. However, it has to be remembered that our study was cross sectional and these five patients represent only the prevalence of undiagnosed atrial fibrillation at a single point in time. Screening would not be appropriate if patients with atrial fibrillation presented with symptoms at the onset of the arrhythmia, but it is not known how often this occurs. Some will undoubtedly present immediately with acute heart failure, rapid palpitations, cardiovascular collapse, or thromboembolism. In others the 
onset may be more insidious, the patient complaining of exertional dyspnoea or fatigue due to chronic heart failure, or even presenting with a chronic brain syndrome due to recurrent cerebral emboli. A proportion will remain asymptomatic despite the arrhythmia and will stay undetected. Data estimating the delay between onset and presentation are important, as this represents a time period without treatment when the patient may suffer unnecessary symptoms and during which a serious and potentially fatal thromboembolic event may occur. Evidence that a significant delay occurs, or that many patients remain asymptomatic after the onset of atrial fibrillation, adds weight to the argument for ECG screening. This information can only be collected in a prospective study, such as we are presently initiating in Sheffield.

Screening for atrial fibrillation may also yield useful information about important associated conditions such as cardiac failure, hypertension, and valvar heart disease. Framingham data $^{20}$ showed that hypertensive heart disease was the most common condition associated with the development of atrial fibrillation, although cardiac failure and rheumatic heart disease have a higher predictive value due to their lower prevalence in the general population.

In our study we found an increased left ventricular mass index in 18 patients $(32.1 \%)$, five occurring in patients with aortic stenosis and one in a patient with hypertrophic cardiomyopathy. Of the remaining 13 patients, all of whom had only mild left ventricular hypertrophy, only three were being treated for hypertension. Conversely, seven of the 10 hypertensive patients had normal left ventricular mass. There remains a group of patients with mild left ventricular hypertrophy in the absence of aortic stenosis, treated hypertension, athleticism, or other features of hypertrophic cardiomyopathy. It is conceivable that this represents a response to a level of blood pressure not felt worthy of treatment using conventional criteria, and perhaps these patients should be considered for 24 hour ambulatory blood pressure monitoring, with a low threshold for treating borderline hypertension. The association with cardiac failure is complex. This condition is the most powerful risk factor for the development of atrial fibrillation, but may also occur as a direct result of the arrhythmia.

Separating cause and effect has important implications for the therapeutic management of the patient. It is likely that the abnormalities in systolic and diastolic function resulting, for example, from ischaemic heart disease and hypertension, predispose to atrial fibrillation by increasing the left ventricular end diastolic pressure and hence the left atrial afterload. Equally, loss of the atrial contribution to ventricular filling (which is particularly important in this age group) may itself precipitate the syndrome of heart failure, even in the presence of a normally contracting ventricle. This latter mechanism of diastolic heart failure is compounded by the abnormalities of left ventricular diastolic function that often coexist in these patients. Identifying the causal mechanism is important as this will direct additional treatment towards left ventricular contractile dysfunction, hypertension, or valvar heart disease.

We noted 18 patients who were being treated with ACE inhibitors or loop diuretics for symptoms suggestive of cardiac failure. Of the 16 patients receiving loop diuretics, only two had a reduced left ventricular ejection fraction, three had mitral stenosis, and two had increased left ventricular mass. This suggests that nine patients $(56 \%)$ were receiving diuretics because of symptoms primarily caused by the arrhythmia itself. Only three patients were receiving ACE inhibitor drugs; one of these had mitral stenosis, one had reduced left ventricular function, and one an increase in left ventricular mass index but no history of hypertension. Conversely, of the 12 patients with reduced left ventricular contractility, only the one patient mentioned above was receiving an ACE inhibitor. While we are well aware of the difficulty in diagnosing underlying left ventricular systolic dysfunction on clinical grounds alone, ${ }^{21}$ this may be particularly apparent in patients with atrial fibrillation because of the associated and confounding influence of abnormalities in diastolic function.

Our study also identified several patients with significant valvar heart disease. As the study did not contain an echocardiographic control group, we do not know for certain whether the number found is in excess of that expected from screening a population of this age. Our own previous data suggest that it is: echocardiographic screening data from two primary care populations of this age ${ }^{2122}$ with suspected heart failure showed substantially lower pick up rates of mitral stenosis $(1.2 \%$ and $2.5 \% v 7.1 \%$ in this study), mitral regurgitation $(12.8 \%$ and $26.7 \%$ v $48.2 \%)$, aortic stenosis $(2.5 \%$ and $4.9 \% v 8.9 \%)$, and left ventricular hypertrophy (measured in one study only, $15.3 \%$ v $32.1 \%$ ). Further controlled studies would, however, be necessary to confirm this finding.

In view of the echocardiographic abnormalities found in this study, should we be recommending cardiac ultrasound in all patients with atrial fibrillation? Our experience from this study is that as far as anticoagulation is concerned, clinical factors alone have by far the greatest influence on the decision making process in the majority of cases. The echocardiographic findings alone rarely altered this decision, although there were notable exceptions. Perhaps a greater role of cardiac ultrasound in this age group is in the identification of associated pathology. In younger patients we accept that many would base the decision to anticoagulate on the presence of associated structural heart disease, although most would agree that such a policy is not applicable to an older population such as this. Overall, we feel that in these patients with atrial fibrillation the single most important risk factor for stroke is the arrhythmia itself. Undoubtedly the best prophylactic treatment is with warfarin and the decision to anticoagulate can be made on the basis of clinical infor- 
mation in the majority of cases. Cardiac ultrasound for all would be ideal, predominantly to address the issue of associated pathology, but with limited resources this is unlikely to be attainable.

The issue of monitoring the increasing numbers of patients taking long term anticoagulants is widely debated. There is concern from primary care physicians over the lack of funding for shifting the burden into the community, which can only be exacerbated by any proposed increase in patient numbers. Adequate funding has to be made available in order that primary care practices can take on this role, and other issues such as near patient testing, quality control, computerised treatment algorithms, ${ }^{23}$ and anticoagulant nurse specialists ${ }^{24}$ have to be addressed if we are to deliver safe and effective thromboembolic prophylaxis to our patients with atrial fibrillation.

We wish to acknowledge the support of the Northern General Hospital Heart Research Fund and the cooperation of Drs J P Grant, B C Metcalfe, and D A Savage, primary care physicians arant, B C Metcalfe, and D A Savage, prin

1 Wheeldon NM. Atrial fibrillation and anticoagulant therapy. Eur Heart 7 1995;16:302-12.

2 Ezekowitz MD Bridges SL, James KE et al for The Veterans Affairs Stroke Prevention in Non-rheumatic Atrial Fibrillation Investigators. Warfarin in the prevention of stroke associated with non-rheumatic atrial fibrillation. $N$ Engl f Med 1992;327:1406-12.

3 Stroke Prevention in Atrial Fibrillation Investigators. Stroke prevention in atrial fibrillation study. Circulation 1991;84: 527-39.

4 Ostrander LD, Brandt RL, Kjelsberg MO, et al. Electrocardiographic findings among the adult population of a total natural community, Tecumseh, Michigan. Circulation 1965;31:888-98.

5 Rose G, Baxter PI, Reid DD, et al. Prevalence and prognosis of electrocardiographic findings in middle-aged men. $B r$ Heart $\mathcal{f} 1978 ; 40: 636-43$.

6 Onundarson PT, Thorgeirsson G, Jomundsson E, et al. Chronic atrial fibrillation-epidemiologic features and 14 year follow-up: a case-control study. Eur Heart $\mathcal{F} 1987 ; 8$ : 521-7.
7 Hill ID, Mottram EM, Killeen PD. Study of the prevalence Hill ID, Mottram EM, Killeen PD. Study of the prevalence years of age. $\mathcal{F}$ R Coll Gen Pract 1987;37:172-3.

8 Wolf PA, Abbott RD, Kannel WB. Atrial fibrillation: a major contributor to stroke in the elderly. The Framingham Study. Arch Intern Med 1987;147:1561-4.

9 Lake FR, Cullen KJ, de Klerk NH, et al. Atrial fibrillation and mortality in an elderly population. Aust NZ $\mathcal{F ~ M e d ~}$ 1989;19:321-6.

10 Chang HI, Bell JR, Deroo DB, et al. Physician variation in anticoagulating patients with atrial fibrillation. Arch Intern Med 1990;150:81-4.

11 Kutner M. Physicians attitudes toward oral anticoagulants and antiplatelet agents for stroke prevention in elderly patients with atrial fibrillation. Arch Intern Med 1991;151: 1950-3

12 Sudlow CM, Rodgers H, Kenny RA, et al. Service provision and use of anticoagulants in atrial fibrillation. $B M F$ 1995;311:558-61.

13 Stroke Prevention in Atrial Fibrillation Investigators. Predictors of thromboembolism in atrial fibrillation: II. Echocardiographic features of patients at risk. Ann Intern Med 1992;116:6-12.

14 The Boston Area Anticoagulation Trial for Atrial Fibrillation Investigators. The effect of low-dose warfarin on the risk of stroke in patients with non-rheumatic atrial fibrillation. $N$ Engl f Med 1990;323:1505-11.

15 Devereux RB, Reichek N. Echocardiographic determination of left ventricular mass in man. Anatomic validation of the method. Circulation 1977;55:613-20.

16 Weyman AK. In: Principles and practice of echocardiography, 2nd ed. Boston: Lea and Febiger, 1994:647-9.

17 The Stroke Prevention in Atrial Fibrillation Investigators. Predictors of thromboembolism in atrial fibrillation: I. Clinical features of patients at risk. Ann Intern Med 1992;116:1-5.

18 Moulton AW, Singer DE, Haas JS. Risk-factors for stroke in patients with non-rheumatic atrial fibrillation: a casecontrol study. Am F Med 1991;91:156-61.

19 Gustafsson C, Asplund K, Britton M, et al. Costeffectiveness of primary stroke prevention in atrial fibrillation: Swedish national perspective. BMf 1992;305: 1457-60.

20 Kannel WB, Abbott RD, Savage DD, et al. Epidemiologic features of chronic atrial fibrillation. $N$ Engl $7 \mathrm{Med}$ 1982;306:1018-22

21 Wheeldon NM, Macdonald TM, Flucker C, et al. Echocardiography in chronic heart failure in the community. $Q \mathcal{F}$ Med 1993;86:17-23.

22 Clarkson PBM, Prasad N, Wheeldon NM, et al. Echocardiographic screening of patients treated with loop diuretics in primary care [abstract]. Amsterdam: Proceedings of Heart Failure '95:101

23 Poller L, Wright D, Rowland SM. Prospective comparative study of computer programmes used for management of warfarin. F Clin Pathol 1993;46:299-303.

24 Mackie C. Nurse practitioners managing anticoagulant clinics. Nursing Times 1996;(3):25-6. 